\title{
INTERNET Y NUEVOS MEDIOS: ESTUDIO SOBRE USOS Y OPINIONES DE LAS PERSONAS MAYORES EN ESPAÑA
}

\author{
Our seniors' challenge to the new media: \\ uses and opinions
}

Mireia Montaña, Elisenda Estanyol y Ferran Lalueza

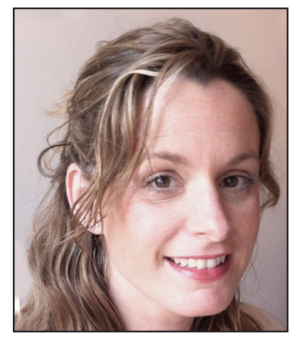

Mireia Montaña es doctora en comunicación por la Universitat Pompeu Fabra (UPF) y licenciada en publicidad y relaciones públicas por la Universitat Ramon Llull. Es profesora de los Estudios de Ciencias de la Información y de la Comunicación en la Universitat Oberta de Catalunya. Es miembro del grupo de investigación GAME - Communication \& New Media (IN3-UOC) y del grupo de investigación Communication, Advertising \& Society (CAS) de la UPF.

http://orcid.org/0000-0002-6464-5129

mmontanabl@uoc.edu

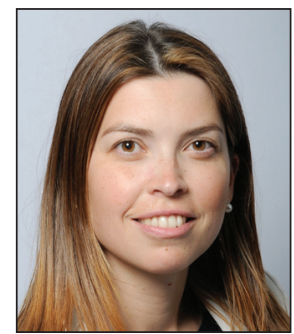

Elisenda Estanyol es doctora en publicidad y relaciones públicas por la Universitat Autònoma de Barcelona (UAB), máster en comunicación empresarial especializado en tecnologías digitales por la Universitat Pompeu Fabra, y licenciada en comunicación audiovisual por la UAB. Es profesora de los Estudios de Ciencias de la Información y de la Comunicación en la Universitat Oberta de Catalunya $(U O C)$ y miembro del grupo de investigación GAME - Communication \& New Media (IN3-UOC).

http://orcid.org/0000-0003-3986-0377

eestanyol@uoc.edu

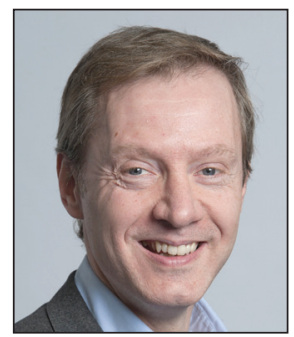

Ferran Lalueza es doctor en periodismo por la Universitat Pompeu Fabra y licenciado en ciencias de la información por la Universitat Autònoma de Barcelona. Es profesor agregado en la Universitat Oberta de Catalunya y coeditor de la revista BiD: Textos universitaris de biblioteconomia $i$ documentació, y dirige COMeIN. Revista de los Estudios de Ciencias de la Información y de la Comunicación desde su fundación. Es miembro del grupo de investigación GAME - Communication \& New Media (IN3-UOC).

http://orcid.org/0000-0002-7010-9795

flalueza@uoc.edu

Universitat Oberta de Catalunya Rambla del Poblenou, 156. 08018 Barcelona, España

\section{Resumen}

Esta investigación persigue determinar el uso mediático que realiza la población mayor de 61 años en España, su relación con las nuevas tecnologías, y sus opiniones. Se han entrevistado 383 individuos residentes en España en el marco de la edición 2013 del World Internet Project (WIP). La muestra ha sido segmentada en dos categorías: young olds (de 61 a 70 años), y middle-old olds (de 71 años en adelante). Los resultados obtenidos han sido comparados con los de la población española en general. Se concluye que, aunque valoran muy positivamente los medios tradicionales, los mayores muestran un considerable apego a internet y más del $60 \%$ comprueba su correo a diario. El uso que hacen de internet es eminentemente pragmático, por lo cual tienden a desaprovechar su potencial como medio de socialización.

\section{Palabras clave}

Población senior; Personas mayores; Tercera edad; Usos de internet; Brecha digital; Medios de comunicación; Videojuegos; World Internet Project; Redes sociales.

\section{Abstract}

This research was aimed at determining the media use by the Spanish population over 61 years, their relation with the new technologies and their opinions. We interviewed 383 Spaniards as part of the 2013 World Internet Project (WIP). The sample 
was segmented into two categories: young olds (from 61-70 years), and middle-old olds (71 and over). The results have been compared to the Spanish general population to detect the most relevant specifities. It can be concluded that, although the assessment they made of traditional media is above average, the Spanish senior population shows considerable interest for internet and more than $60 \%$ of them check their email daily. Their use of internet, however, is eminently pragmatic, so they tend to not use its potential as a socialization media.

\section{Keywords}

Senior people; Aged people; Elderly; Older consumers; Internet uses; Digital divide; Media; Video games; World Internet Project; Social networks.

Montaña, Mireia; Estanyol, Elisenda; Lalueza, Ferran (2015). "Internet y nuevos medios: estudio sobre usos y opiniones de los seniors en España". El profesional de la información, v. 24, n. 6, pp. 759-765.

http://dx.doi.org/10.3145/epi.2015.nov.07

\section{Introducción}

Internet ha permitido establecer una nueva forma de comunicarse habitual e imprescindible para buena parte de la población. No todos los segmentos de población utilizan internet con la misma intensidad, siendo el uso menor cuanto mayor es el individuo. Este artículo tiene como objetivo analizar los principales usos y opiniones que tienen las personas mayores en España sobre los nuevos medios e internet. Los mayores de 60 años representan un 25\% de la población española $(I N E, 2014)$ y son el grupo de población que más crece debido al aumento de la esperanza de vida y a la disminución de la natalidad, lo que se ha bautizado como senior boom. Debido a la importancia de este grupo se considera relevante investigar cómo interactúa con internet y los videojuegos, pues hasta la fecha han sido pocos los estudios sobre este tema.

Este artículo tiene como objetivos específicos:

- conocer la valoración de los nuevos medios como fuente de información y entretenimiento por parte de la población mayor en España;

- comparar esta valoración con la de los medios de comunicación tradicionales como TV, radio y prensa;

- determinar la frecuencia de uso de internet entre la población mayor comparándola con el total de la población;

- analizar el tipo de uso de internet entre los seniors;

- realizar una comparativa de usos y valoraciones subdividiendo la población mayor entre young olds (YO) y middle old-olds (MO).

El conocimiento de las prácticas y opiniones de la tercera edad puede ser de utilidad para diversos sectores como el sanitario, el publicitario o el comercial. Tanto las administraciones públicas como las empresas pueden implementar estrategias de comunicación dirigidas a este segmento.

\section{Estado de la cuestión}

En España, los informes anuales que realizan las fundaciones de diversas compañías telefónicas ayudan a perfilar un mapa del uso y penetración de internet (Fundación Telefónica, 2013; Fundación Vodafone, 2011; 2012; Fundación Orange, 2014).

Asimismo, desde organismos públicos como Ontsi (2014; 2013), Imserso (2011) y el Instituto Nacional de Estadística
(2014) afloran anualmente datos relevantes para conocer cómo se está implantando internet en la población española.

Así, se conoce que el número de españoles que accede a internet continúa incrementándose (la penetración de internet alcanzó el $71,6 \%$ en 2013 , según el INE), aunque lo hace de forma desigual en función de la edad. Así, mientras que casi el $98 \%$ de los jóvenes accede a internet, este porcentaje disminuye de forma importante a medida que crece la edad, de forma que sólo el $18 \%$ de los mayores de 65 años se conecta a la Red (INE, 2013). Este dato, además, debe contextualizarse teniendo en cuenta que España:

"aún se encuentra por detrás de muchos países europeos en relación con la frecuencia con la que los mayores de 65 años se conectan a internet con regularidad" (Sebastián-Morillas; Martínez-Navarro, 2013, p. 1).

\subsection{Mayores e internet}

Se ha bautizado como fobia online el rechazo a las nuevas tecnologías experimentado, sobre todo, por parte de la población mayor. Este rechazo, además, se ha detectado en mayor medida en aquellas personas que han llegado a la madurez en un entorno sin internet (Reisenwitz et al., 2007, p. 408). Las limitaciones físicas y cognitivas que afectan a algunos de los individuos de mayor edad, también influyen en la aversión a las nuevas tecnologías (Fundación Orange, 2014 , p. 106). Sin embargo, hay informes que señalan que la actitud de los mayores frente a las nuevas tecnologías puede adaptarse, y que se muestran receptivos cuando van acompañados de un entorno confortable y de una formación adecuada (Kiel, 2005, p. 21).

Fijándonos concretamente en la navegación web, se ha identificado que las tipografías demasiado pequeñas, los pop-ups y el exceso de aplicaciones flash dificultan la usabilidad para los mayores (Pepper, 2002), por lo que recomiendan que las webs dirigidas a ellos sean más claras e intuitivas.

El hecho de que parte de este grupo de la población no disponga en sus hogares de ordenador, tablet o smartphone dificulta también su uso de internet. Centros públicos, tales como bibliotecas o asociaciones de tercera edat, equipados con ordenadores con conexión a internet, ayudan a reducir estas limitaciones. 
Los beneficios que internet puede aportar a las personas mayores han sido detallados en numerosos estudios, desde las facilidades para comprar y pagar recibos hasta la posibilidad de aprender y estar en contacto con otras personas (Lawhon; Ennis; Lawhon, 1996; Adler, 2002; Campbell, 2008; Chen, 2008; McMellon; Schiffman, 2000).

Las personas mayores gozan de un estilo de vida muy diferente al de anteriores generaciones; el aumento de la esperanza de vida, que en España es de 79,4 años para los hombres y de 85,1 años para las mujeres (INE, 2014), y una condición más saludable les abren nuevas oportunidades para mantenerse activos socialmente (Imserso, 2011; Sebastián-Morillas; Martínez-Navarro, 2013, p. 3). En este contexto, internet posibilita acceder a información que les resulta de interés y utilidad, acceder a ofertas comerciales y culturales, a la vez que puede convertirse en una ayuda para realizar trámites sanitarios y administrativos.

Como constatan Marín-Díaz y García-Fernández (2003, p. 125), internet está influyendo en el establecimiento de modos y maneras de lenguaje emergentes, así como nuevas formas de establecer vínculos. En este sentido, cabe recordar que las comunicaciones sociales son un factor esencial para la calidad de vida, que tiende a disminuir con la edad. Las tecnologías posibilitan a los mayores comunicarse con sus familiares y amigos, participar en debates y materias de interés, reducir el aislamiento social y el distanciamiento intergeneracional, y ser fuente de entretenimiento. Asimismo, internet se constituye como una forma de acceder a la formación continua o lifelong learning (Lee; Yiwei; Hewitt, 2011; Bond et al., 2010; Slegers; Van-Boxtel; Jolles, 2008).

El uso de internet entre la población de edad más avanzada se prevé que se incremente en el futuro, puesto que las nuevas generaciones de mayores de 65 años cada vez tendrán más formación.

\subsection{Descripción y clasificación de los seniors}

Dado que el análisis se focaliza en los seniors, se revisan a continuación algunas de las clasificaciones y descripciones propuestas por varios autores.

Tradicionalmente se define la población mayor como la de más de 65 años (Eastman; Iyer, 2004), pero algunos autores han propuesto subdividir esta gran franja de edad acuñando conceptos como young olds, old olds y oldest olds (Fisher, 1993; Atchley; Barusch, 2004; Austrian, 2008; Lee; Yiwei; Hewitt, 2011; Schaie, 2012). Otros autores amplían el grupo de mayores agregando aquellos adultos de entre 50 y 64 años, que denominan pre-seniors o pre-jubilados (Whitford, 1998).

Más allá de su clasificación cronológica, otros autores han ahondado en el estudio psicológico y sociológico de este grupo poblacional teniendo en cuenta las motivaciones, necesidades e intereses de los individuos (Huberman, 1974; Levinson, 1978; Amador-Muñoz et al., 2001). Por su parte, Havighurst (1978) describió a los mayores de 65 años como etapa de "segunda madurez" apuntando que entre las dificultades con las que se encuentran están: la adaptación a la jubilación y a la disminución de los ingresos, la aceptación de la muerte de conocidos -y puede que del cónyuge-, la adhesión a grupos de su edad y la adaptación a una forma de vida que les posibilite hacer frente a las deficiencias físicas.

\section{Material y métodos}

La investigación realizada forma parte del informe WIP España 2013. La técnica empleada es CATI Bellview, una entrevista telefónica asistida por ordenador a individuos de 16 y más años, residentes en hogares españoles con líneas de teléfono fijas. Se realizaron 28.021 llamadas para obtener las 1.600 entrevistas de la muestra, obteniendo una tasa de respuesta del 5,71\%. El trabajo de campo se realizó entre los días 2 y 20 de diciembre de 2013. La muestra está segmentada por comunidades autónomas, de modo proporcional a la distribución real de la población. A partir del censo se construyó la muestra en base a cuotas representativas de la población española. Se utilizó una base de datos pública con los teléfonos fijos existentes y se realizaron las entrevistas según las cuotas establecidas.

\section{El uso de la Red que realizan los mayores es más práctico que social}

Las unidades primarias de provincia (núcleos de población) han sido seleccionadas de forma aleatoria proporcional para cada provincia.

Las unidades secundarias (hogares) se han seleccionado aleatoriamente a partir de los números telefónicos.

Las unidades últimas (individuos) han sido elegidas mediante una estratificación cruzada de sexo, edad y tamaño de municipio (éste se subdivide en 7 tipos de hábitats según su dimensión).

Además de las anteriores, también se tuvo en cuenta la variable sociodemográfica del paro. El margen de error para el total de la muestra es de $+/-2,45 \%$, para $p=q=50 \%$ y bajo el supuesto de máxima indeterminación.

Tomando como sistema de clasificación de los mayores por edad la propuesta recogida en Barrouillet y Gaillard (2010), se ha agrupado la muestra en dos grandes grupos:

- los young olds (YO): de 61 a 70 años;

- los middle-old olds (MO): de 71 años en adelante.

Así, la muestra resultante ha sido de 301 individuos en la franja de YO y de 82 en la franja de MO. El margen de error para esta nueva muestra de individuos de 61 y más años es de $+/-4,9 \%$, para $p=q=50 \%$ y bajo el supuesto de máxima indeterminación.

Las respuestas obtenidas con este colectivo se han comparado con la media de la población para extraer información significativa sobre el uso que hace la población senior de los medios tradicionales y los nuevos medios, internet y videojuegos en concreto, así como la opinión que les merecen. Para el análisis se ha realizado una tabla con todas las respuestas del cuestionario y éstas se han cruzado con todas las variables sociodemográficas. 


\section{Análisis y resultados}

Tanto los YO como los MO dan una trascendencia informativa a internet similar al resto de la población (alrededor del $78 \%)$.

Aunque están por debajo de la media española, ambos grupos utilitzan internet de forma habitual. Comprueban su correo electrónico a diario en un porcentaje del $69 \%$ y del $58 \%$ respectivamente.

Es destacable el uso que realiza la gente mayor de internet para leer noticias, con un porcentaje muy similar a la media de la población, alrededor del 58\%. Si comparamos la consideración que tienen los seniors de los medios de comunicación más tradicionales vemos que la televisión está muy valorada como fuente informativa y como entretenimiento para el segmento de los $\mathrm{MO}$ (69\% y un $72 \%$ respectivamente).

La prensa como soporte informativo toma mucha trascendencia, sobre todo a partir de los 71 años (67\%). Aunque también la valoran como fuente de entretenimiento, lo hacen en menor porcentaje (45\%).

Los $\mathrm{MO}$ son quienes atribuyen mayor repercusión a la radio como medio informativo (78\%; 19,2 puntos por encima de la media española). También la valoran como fuente de entretenimiento (57\%).

\section{El uso de la mensajería instantánea va decreciendo a medida que aumenta la edad del entrevistado}

Aunque con menor asiduidad que el total de la población, vemos que los mayores, sobre todo los YO, también utilizan la Red para buscar información sobre productos o cuestiones relacionadas con la salud (24\%). Una de las informaciones que más buscan sobre los productos son sus precios, para poderlos comparar. $Y$ uno de los productos sobre los que buscan información habitualmente son los viajes. Aunque los YO realizan un uso similar al del resto de la población (41\%), este porcentaje decrece significativamente en el caso de los $\mathrm{MO}(22 \%)$.

Una de las alternativas de internet que utilizan en menor proporción que la media son las redes sociales y la mensajería instantánea tipo Skype o Whatsapp. Las redes sociales son utilizadas en un $19 \%$ y un $6 \%$ entre los YO y los MO respectivamente, ante el $40 \%$ de la media de la población. En cambio, los resultados obtenidos constatan que los vínculos interpersonales presenciales sí son valorados como fuente de información de manera creciente según avanza la edad de la persona entrevistada.

Los YO utilizan la mensajería instantánea en un porcentaje del $49 \%$, cifra que decrece hasta el $22 \%$ para los $\mathrm{MO}$. El uso va decreciendo a medida que aumenta la edad del entrevistado. A este colectivo tampoco le gusta crear contenido propio ni re-publicar o compartir links de otros.

Aunque hay seniors que compran por internet (el 18\% de los YO y el $13 \%$ los MO) ésta no es la propensión. Respecto a la realización de reservas de viajes online, sin embargo, se aprecia que los YO no están demasiado por debajo de la media de la población (un 14\% para los YO ante un $16 \%$ de la media española). En el caso de los middle-old olds, ya decrece a un $6 \%$.

Aunque se sitúan por debajo de la media de la población (50\%), se aprecia que la gente mayor utiliza habitualmente la banca online. Así lo afirman el $43 \%$ de los YO y el $38 \%$ de los middle y old olds, que aseguran conectarse al menos una vez al mes.

Por último, prácticamente la mitad de la población mayor de 60 años considera que el juego es una forma natural de aprender. Un tercio de los middle-old olds afirma que con los videojuegos se aprenden cosas útiles. Éstos también son valorados por los mayores como fuente para favorecer la memoria y la atención. Aunque ven aspectos positivos en los videojuegos, muchos también opinan que crean nerviosismo, adicción, aislamiento o que pueden provocar conductas violentas.

\section{Discusión y conclusiones}

Está ampliamente extendida la creencia de que las personas mayores están apartadas de internet. Sin embargo, los resultados de la presente investigación constatan que aun siendo el grupo de la población que más lentamente se está incorporando a la misma, hacen un uso considerable de ella. Es previsible que el uso aumente a medida que las nuevas generaciones de mayores lleguen a la vejez, estando ya cada vez más familiarizadas con internet y las redes sociales.

Aunque los mayores de 61 años siguen valorando los medios tradicionales, como televisión, prensa y radio, y lo hacen por encima de la media de la población española, es destacable el peso que dan a internet. De hecho, tanto los young olds (YO) como los middle-old olds ( $\mathrm{MO}$ ) valoran internet en un porcentaje equiparable al de la media de la población. Comprueban su correo electrónico a diario en un $69 \%$ y un $58 \%$ respectivamente. Esta conclusión contrasta con la visión pesimista aportada por la Fundación Orange (2014, p. 7), cuyo informe apunta a que las personas con mayor dificultad para acceder a internet son los colectivos con niveles educativos bajos o con rentas bajas, las personas mayores de 55 años, las personas con discapacidad, quienes habitan en zonas rurales y la población inmigrante, siendo de todos ellos el colectivo de las personas mayores el que presenta aún dificultades más destacadas, y resultando además la exclusión digital en España más grave que en el resto de Europa.

En cambio, está en sintonía con los datos aportados por el Observatorio Nacional de Telecomunicaciones y de la Sociedad de la Información, que constata que la población que ahora tiene entre 55 y 64 años constituye el 15,2\% de los internautas españoles, mientras que un $11,5 \%$ lo integran las personas que tienen entre 65 y 74 años. Entre los equipos utilizados en el hogar para acceder a internet, el más frecuente es el teléfono móvil, desde el que se conecta el $80,7 \%$, seguido del ordenador portátil con el 69,3\% y del ordenador de sobremesa con el 62,4\% (Ontsi, 2014). 
Tabla 1. Comparativa de la valoración y el uso de los medios de comunicación segmentada por los públicos estudiados

\begin{tabular}{|c|c|c|c|c|c|c|}
\hline & $\begin{array}{c}\text { Total } \\
\text { población }\end{array}$ & YO & I.A. & MO & İ.A. & $\begin{array}{l}\text { Í.A. YO } \\
\text { vs MO }\end{array}$ \\
\hline \multicolumn{7}{|l|}{ \% que utiliza internet 1 ó más veces al día para... } \\
\hline Buscar o navegar & 65 & 44 & 6 & 32 & 49 & 139 \\
\hline Comprobar mail & 73 & 69 & 9 & 58 & 80 & 118 \\
\hline Mensajería instantánea (Skype, Whatsapp...) & 68 & 49 & 7 & 22 & 32 & 226 \\
\hline Leer noticias nacionales o internacionales & 58 & 56 & 9 & 58 & 100 & 97 \\
\hline \multicolumn{7}{|l|}{ \% que utiliza internet 1 ó más veces a la semana para... } \\
\hline Visitar redes sociales & 40 & 19 & 47 & 6 & 15 & 308 \\
\hline Publicar contenido que crea uno mismo (vídeos, fotos, escritos...) & 25 & 10 & 38 & 10 & 38 & 100 \\
\hline Re-publicar, compartir links o contenido creado por otros & 32 & 19 & 59 & 10 & 30 & 199 \\
\hline Buscar información relacionada con la salud & 28 & 24 & 87 & 22 & 79 & 110 \\
\hline $\begin{array}{l}\text { Conseguir información sobre un producto (coche, libro, tecnolo- } \\
\text { gía...) }\end{array}$ & 46 & 33 & 71 & 25 & 55 & 131 \\
\hline Escuchar radio online & 24 & 14 & 61 & 9 & 40 & 153 \\
\hline \multicolumn{7}{|l|}{ \% que utiliza internet 1 ó más veces al mes para... } \\
\hline Leer blogs & 43 & 32 & 75 & 19 & 45 & 168 \\
\hline Comparar precios de productos/servicios & 51 & 31 & 62 & 16 & 31 & 200 \\
\hline Comprar online & 32 & 18 & 57 & 13 & 40 & 144 \\
\hline Buscar información sobre un viaje & 43 & 41 & 95 & 22 & 51 & 185 \\
\hline Hacer reservas o comprar viajes & 16 & 14 & 83 & 6 & 38 & 219 \\
\hline Usar banca online & 50 & 43 & 87 & 38 & 77 & 113 \\
\hline \multicolumn{7}{|l|}{$\begin{array}{l}\text { Valoración de videojuegos } \\
\% \text { que está de acuerdo o muy de acuerdo con... }\end{array}$} \\
\hline Aprender a través del juego es una forma natural de aprender & 45 & 47 & 104 & 46 & 102 & 102 \\
\hline Con los videojuegos se aprenden cosas útiles & 19 & 18 & 95 & 33 & 174 & 55 \\
\hline Los videojuegos favorecen la memoria y la atención & 54 & 63 & 117 & 60 & 111 & 105 \\
\hline Los videojuegos permiten liberar el estrés & 41 & 38 & 93 & 45 & 110 & 84 \\
\hline Los videojuegos se juegan mejor en compañía de otros & 53 & 43 & 81 & 60 & 113 & 72 \\
\hline \multicolumn{7}{|l|}{$\begin{array}{l}\text { Valoración de los medios de comunicación } \\
\% \text { que cree que es importante o muy importante... }\end{array}$} \\
\hline La TV como fuente de información & 40 & 42 & 103 & 69 & 170 & 61 \\
\hline La TV como entretenimiento & 52 & 49 & 96 & 72 & 140 & 68 \\
\hline La prensa como fuente de información & 53 & 5 & 97 & 67 & 126 & 76 \\
\hline La prensa como entretenimiento & 31 & 37 & 118 & 45 & 144 & 82 \\
\hline La radio como fuente de información & 59 & 61 & 104 & 78 & 133 & 78 \\
\hline La radio como entretenimiento & 53 & 57 & 108 & 57 & 108 & 100 \\
\hline Internet como fuente de información & 78 & 77 & 99 & 78 & 99 & 100 \\
\hline Internet como entretenimiento & 63 & 49 & 78 & 67 & 106 & 74 \\
\hline \multicolumn{7}{|l|}{$\begin{array}{l}\text { Valoración de las relaciones } \\
\% \text { que cree que son importantes o muy importantes... }\end{array}$} \\
\hline Las relaciones interpersonales como fuente de información & 58 & 63 & 108 & 65 & 112 & 96 \\
\hline
\end{tabular}

I.A. Índice de afinidad. Establece la relación entre la penetración del medio respecto del público objetivo (mayores) y la penetración de este medio respecto del total del universo (total España). Los índices que superan el valor de 100 señalan un buen nivel de afinidad y los que están por debajo de 100, en cambio, se consideran poco afines. Se establece según la fórmula:

Índice de afinidad $=\quad$ Penetración del medio en el público objetivo

Penetración del medio en el universo total

Desde esta perspectiva, se confirma que el potencial que tiene internet para interactuar con los ciudadanos que están en la tercera edad no está siendo aprovechado (Trocchia; Janda, 2000, p. 605). Un ejemplo sería que sólo el $5 \%$ de la publicidad online se destina a este colectivo (Nielsen, 2014), estando muy por debajo no sólo del peso real que tiene en el conjunto de la población (30\%) sino también del uso frecuente y la valoración positiva que los mayores hacen de internet, así como de la mayor disponibilidad de tiempo para navegar en la Red. 
Como factor diferencial, el uso de la Red que realizan los mayores es más práctico que social. Son grandes lectores de periódicos online, utilizan servicios de banca, buscan información sobre productos, comparan precios y además, en el caso de los YO, reservan viajes en un porcentaje similar a la media española.

En cambio, las alternativas más sociales que proporciona internet no son tan valoradas. Utilizan las redes sociales muy por debajo de la media, sobre todo los MO. Dada la constatación de que el uso va decreciendo a medida que aumenta la edad del individuo, puede deducirse que la población mayor no se siente identificada con las redes sociales ni percibe el potencial que éstas tienen para mantenerles en contacto con sus allegados. Esta conclusión tiene plena consistencia con la idea de que la jubilación reduce la vida social (Huberman, 1974; INE, 2012) y con la constatación de que la edad es directamente proporcional a la valoración que hacemos de la interacción presencial.

\section{Los mayores no aprovechan el potencial} que tiene internet para interactuar

Este patrón de comportamiento en el que lo pragmático prima sobre lo social contrasta con los patrones de comportamientos de la población en general, con una utilización de internet en la que ambas finalidades se entremezclan de forma menos diferenciada. Así, según datos del INE (2014):

- 70,2\% de la población española usa internet para leer noticias online;

- 66,5\% busca información sobre bienes y servicios;

- 64,8\% busca información sobre educación y formación;

- 64,1\% participa en redes sociales;

- $62,1 \%$ accede a enciclopedias online;

- $61 \%$ busca información sobre materias de salud;

- 52,7\% utiliza servicios de viajes;

- 36,3\% descarga software;

- 28,4\% telefonea por internet o realiza videollamadas;

- 27,4\% busca empleo o envía currículos;

- $11,8 \%$ utiliza internet para vender bienes o servicios.

Finalmente, puede concluirse también que la valoración que reciben los videojuegos por parte de los mayores de 60 años es más positiva de lo esperado a partir de los tópicos generalmente asumidos, ya que prácticamente la mitad de este colectivo considera que el juego es una forma natural de aprender. Los videojuegos también son valorados por este colectivo como plataforma que favorece la memoria y la atención, en un porcentaje superior a la media de la población. A partir de estos datos, se desprende el potencial de los videojuegos entre la gente mayor que, contrariamente a lo que podría parecer, no es reticente a su uso y además valora sus posibilidades. El apego a los juegos, al entretenimiento y a las recompensas de carácter inmediato ya fue atribuido por Huberman (1974) a los mayores de 60 años, y esta conclusión evidencia su vigencia.

Dado que el presente trabajo se ha centrado exclusivamente en el caso español, se considera que como investigación futura podría resultar igualmente productivo y revelador estable- cer comparaciones con otros países, aunque dada la rapidez de los cambios sociales, las mediciones deberían ser simultáneas. Los resultados obtenidos brindan una imagen fija del uso actual de internet entre los seniors, pero el hecho de que la penetración de internet se incremente año a año y que se produzca en un entorno dinámico, anima a proponer igualmente futuras líneas de investigación basadas en la comparativa diacrónica de estos datos con los que se puedan obtener en los próximos años. Por último, la metodología cuantitativa aplicada ha hecho posible extraer datos de uso y valoración -por parte de los seniors - de internet, las redes sociales y los videojuegos; datos que también podrían enriquecerse en el futuro con investigaciones cualitativas que posibiliten profundizar en sus motivos y consecuencias.

\section{Bibliografía}

Adler, Richard (2002). "The age wave meets the technology wave: Broadband and older Americans". SeniorNet. http://www.seniornet.org/downloads/broadband.pdf

Amador-Muñoz, Luis; Monreal-Gimeno, María-del-Carmen; Marco-Macarro, María-José (2001). "El adulto: etapas y consideraciones para el aprendizaje". Eúphoros, n. 3, pp. 97-112. http://dialnet.unirioja.es/servlet/articulo?codigo=1183063

Austrian, Sonia G. (2008). Developmental theories through the life cycle. New York: Columbia University Press. ISBN: 9780231139717

Atchley, Robert; Barusch, Amanda (2004). Social forces and aging: An introduction to social gerontology. Belmont (CA): Wadsworth/Thomson Learning. ISBN: 9780534533450

Barrouillet, Pierre; Gaillard, Vinciane (2010). Cognitive development and working memory: A dialogue between neoPiagetian theories and cognitive approaches. Sussex: Psychology Press. ISBN: 9781848720367

Bond, Gail E.; Burr, Robert; Wolf, Frederic M.; Feldt, Karen (2010). "The effects of a web-based intervention on psychosocial well-being among adults aged 60 and older with diabetes - a randomized trial". The diabetes educator, v. 36, n. 3, pp. 446-456.

http://www.ncbi.nlm.nih.gov/pubmed/20375351

http://dx.doi.org/10.1177/0145721710366758

Campbell, Robert-James (2008). “Meeting seniors' information needs: Using computer technology". Home health care management \& practice, v. 20, n. 4, pp. 328-335. http://dx.doi.org/10.1177/1084822307310765

Chen, Su-Yen. (2008). "Reading practices and profiles of older adults in Taiwan". Educational gerontology, v. 34, n. 5, pp. 427-441.

http://dx.doi.org/10.1080/03601270701834059

Eastman, Jacqueline K.; Iyer, Rajesh (2004). "The elderly's uses and attitudes towards the Internet". Journal of consumer marketing, v. 21, n. 3, pp. 208-220.

http://dx.doi.org/10.1108/07363760410534759

Fisher, James C. (1993). "A framework for describing developmental change among older adults". Adult education quarterly, v. 43, n. 2, pp. 76-89.

http://dx.doi.org/10.1177/0741713693043002002 
Fundación Orange (2014). eEspaña 2014. Informe anual sobre el desarrollo de la sociedad de la información en España. Fundación Orange.

http://fundacionorange.es/fundacionorange/analisis/ eespana/e_espana14.html

Fundación Telefónica (2013) "La Sociedad de la información en España 2013"

http://www.fundaciontelefonica.com/arte_cultura/ publicaciones-listado/pagina-item-publicaciones/?itempubli=261

Fundación Vodafone (2011). Los mayores ante las TIC: accesibilidad y asequibilidad. Fundación Vodafone España; Ministerio de Industria, Turismo y Comercio.

http://www.fundacionvodafone.es/publicacion/losmayores-ante-las-tic-accesibilidad-y-asequibilidad

Fundación Vodafone (2012). TIC y mayores: conectados al futuro. Fundación Vodafone España.

http://www.fundacionvodafone.es/publicacion/tic-ymayores-conectados-al-futuro

Havighurst, Clark C. (1978). "Health maintenance organizations and the health planners". Utah law review, pp. 123-154. http://scholarship.law.duke.edu/cgi/viewcontent.cgi?article $=1347 \&$ context=faculty_scholarship

Huberman, Michael (1974). "Looking at adult education from the perspective of the adult life cycle". International review of education, v. 20, n. 2, pp. 117-137.

http://dx.doi.org/10.1007/BF00598497

Imserso (2011). Envejecimiento activo. Libro blanco. Ministerio de Sanidad, Política Social e Igualdad; Instituto de Mayores y Servicios Sociales (Imserso).

http://www.imserso.es/InterPresent2/groups/imserso/ documents/binario/8088_8089libroblancoenv.pdf

Instituto Nacional de Estadística (INE) (2012). Boletín sobre envejecimiento activo.

http://www.ine.es

Instituto Nacional de Estadística (INE) (2014). El comercio electrónico y el uso de las nuevas tecnologías.

http://www.ine.es

Kiel, Joan M. (2005). "The digital divide: internet and e-mail use by the elderly". Informatics for health and social care, v. 30, n. 1, pp. 19-23.

http://dx.doi.org/10.1080/14639230500066900

Lawhon, Tommie; Ennis, Demetria; Lawhon, David C. (1996). "Senior adults and computers in the 1990s". Educational gerontology: An international quarterly, v. 22, n. 2, pp. 193-201. http://dx.doi.org/10.1080/0360127960220205

Lee, Bob; Chen, Yiwei; Hewitt, Lynne (2011). “Age differences in constraints encountered by seniors in their use of computers and the internet". Computers in human behavior, v. 27, n. 3, pp. 1231-1237.

http://dx.doi.org/10.1016/j.chb.2011.01.003

Levinson, Daniel (1978). The seasons of a man's life. New York: Ballantine. ISBN: 9780345339010
Marín-Díaz, Verónica; García-Fernández, María-Dolores (2003). "La familia e internet, ¿̇un juego a tres bandas?". Comunicar, n. 21, pp. 123-126.

http://goo.gl/16bCMI

McMellon, Charles A.; Schiffman, Leon G. (2000). "Cybersenior mobility: why some older consumers may be adopting the internet". Advances in consumer research, v. 27, pp. 139-144.

http://www.acrwebsite.org/search/view-conferenceproceedings. aspx? $? / d=8377$

Nielsen (2014). Nielsen digital report 2014. http://www.slideshare.net/tinhanhvy/the-digitalconsumer-report-2014-nielsen

http://www.nielsen.com/us/en/insights/reports/2014.html

Observatorio Nacional de Telecomunicaciones y de la Sociedad de la Información (Ontsi) (2013). Perfil sociodemográfico de los internatutas. Análisis de datos INE 2012.

http://www.ontsi.red.es/ontsi/es/estudios-informes/perfilsociodemogr\%C3\%A1fico-de-los-internautas-datos-ine-2012

Observatorio Nacional de Telecomunicaciones y de la Sociedad de la Información (Ontsi) (2014). XLIII oleada del panel hogares: Las TIC en los hogares españoles.

http://goo.gl/2iSNm5

Pepper, Jeff (2002). "Wired and retired: assisted living residents go online". Nursing homes: Long terme care management, n. 51, n. 10, pp. 60-65.

Reisenwitz, Tim; Iyer, Rajesh; Kuhlmeier, David B.; Eastman, Jacqueline K. (2007). "The elderly's Internet usage: An updated look". Journal of consumer marketing, n. 24, pp. 406-418.

http://dx.doi.org/10.1108/07363760710834825

Schaie, K. Warner (2012). Developmental influences on adult intelligence: The Seattle longitudinal study. Oxford: Oxford University Press. ISBN: 9780195386134

Sebastián-Morillas, Ana; Martínez-Navarro, Gema (2013). "La influencia de las nuevas tecnologías: videojuegos, redes sociales e internet, en los consumidores seniors en España". En: I Congreso intl comunicación y sociedad. UNIR, Logroño. ISBN: 9788415626428

http://reunir.unir.net/handle/123456789/1749

Slegers, Karin; Van-Boxtel, Martin P. J.; Jolles, Jelle (2008). "Effects of computer training and internet usage on the wellbeing and quality of life of older adults: A randomized, controlled study". The journals of gerontology series B: Psychological sciences and social sciences, v. 63, n. 3, pp. 176-184. https://atmire.com/dspace-labs3/bitstream/handle/123456789/6930/ file14205.pdf?sequence=1

Trocchia, Philip. J.; Janda, Swinder (2000). “A phenomenological investigation of Internet usage among older individuals". Journal of consumer marketing, v. 17, n. 7, pp. 605-616. https://goo.gl/YWWTNg

Whitford, Marty (1998). "Market in motion". Hotel and motel management, v. 213, n. 7, pp. 41-43. 